\title{
A DIFFERENTIAL OPERATOR FOR SYMMETRIC FUNCTIONS AND THE COMBINATORICS OF MULTIPLYING TRANSPOSITIONS
}

\author{
I. P. GOULDEN
}

\begin{abstract}
By means of irreducible characters for the symmetric group, formulas have previously been given for the number of ways of writing permutations in a given conjugacy class as products of transpositions. These formulas are alternating sums of binomial coefficients and powers of integers. Combinatorial proofs are obtained in this paper by analyzing the action of a partial differential operator for symmetric functions.
\end{abstract}

\section{INTRODUCTION}

Let $\lambda=\left(\lambda_{1}, \lambda_{2}, \ldots\right)$ where $\lambda_{1} \geq \lambda_{2} \ldots$ are nonnegative integers and $\lambda_{1}+$ $\lambda_{2}+\cdots=n$. Then $\lambda$ is a partition of $n$, denoted $\lambda \vdash n$. If $m$ of the $\lambda_{i}$ are positive we also write $\lambda=\left(\lambda_{1}, \ldots, \lambda_{m}\right)$, and say that $\lambda$ has $m$ parts, denoted by $l(\lambda)=m$. If $k_{j}$ of the parts of $\lambda$ are equal to $j$ for $j \geq 1$, we can write $\lambda=1^{k_{1}} 2^{k_{2}} \ldots$.

Associated with every permutation $\sigma$ in $S_{n}$, the symmetric group on $\{1, \ldots, n\}$, is the partition of $n$ whose parts specify the lengths of the cycles in the disjoint cycle representation of $\sigma$. This partition, denoted by $d(\sigma)$, is called the cycle distribution of $\sigma$. For $\alpha \vdash n$, the set of all permutations in $S_{n}$ with cycle distribution $\alpha$ is a conjugacy class, denoted by $\mathscr{C}_{\alpha}$. If $K_{\alpha}$ is the formal sum of the elements of $\mathscr{C}_{\alpha}$, then the set $\left\{K_{\alpha} \mid \alpha \vdash n\right\}$ is a basis for the centre of the group algebra $\mathbb{C} S_{n}$. Thus we can linearize the product of any elements in this set, so if $\alpha_{i} \vdash n$ for $i=1, \ldots, m$ we can write

$$
K_{\alpha_{1}} \cdots K_{\alpha_{m}}=\sum_{\gamma \vdash n} c_{\alpha_{1}, \ldots, \alpha_{m}}^{\gamma} K_{\gamma},
$$

and the numbers $c_{\alpha_{1}, \ldots, \alpha_{m}}^{\gamma}$ are called connection coefficients for the symmetric group.

An expression for the arbitrary connection coefficient can be given as a summation over partitions of $n$, involving characters of the symmetric group. In general this is not very useful because characters are hard to evaluate and the summation set is large. However in certain cases this expression can be reduced to yield a nice explicit form, as a single-indexed binomial sum. Character theory has been used by Stanley [19] and Jackson [13, 14, 15] to give classes of such explicit forms for various connection coefficients involving $n$-cycles (cycle distribution $n$ ) and transpositions (cycle distribution $1^{n-2} 2$ ).

Received by the editors June 10, 1991.

1991 Mathematics Subject Classification. Primary 05A15; Secondary $05 E 05$. 
The form of these results suggests that there should exist derivations of the nice expressions for connection coefficients that are free of the use of characters. In this direction, Bertram and Wei [2], Boccara [3], and Walkup [20] have obtained results for the product of two $n$-cycles by elementary means. Goulden and Jackson [9] have given a direct combinatorial derivation for the value of $c_{\alpha_{1}, \ldots, \alpha_{m}}^{(n)}$ in the case that $l\left(\alpha_{1}\right)+\cdots+l\left(\alpha_{m}\right)=n+1$. Moszkowski [18] has given a direct combinatorial derivation for the value of $c_{\alpha_{1}}^{(n)}, \ldots, \alpha_{n-1}$ in the case that $\alpha_{1}, \ldots, \alpha_{n-1}$ are transpositions (see also Goulden and Pepper [10]).

Bédard and Goupil [1] had previously shown by inductive means that

$$
c_{\alpha_{1}, \alpha_{2}}^{(n)}=\frac{n\left(l\left(\alpha_{1}\right)-1\right) !\left(l\left(\alpha_{2}\right)-1\right) !}{\prod_{j \geq 1} \beta_{1 j} ! \beta_{2 j} !}
$$

where $\alpha_{i}=1^{\beta_{i 1}} 2^{\beta_{i 2}} \cdots$ for $i=1,2$, and $l\left(\alpha_{1}\right)+l\left(\alpha_{2}\right)=n+1$. In the case $m=2$ of Goulden and Jackson [9], the RHS of (1.1) is shown to be the number of two-coloured plane trees of a certain type, and an explicit bijection is given between these trees and pairs of permutations $(\sigma, \rho) \in \mathscr{C}_{\alpha_{1}} \times \mathscr{C}_{\alpha_{2}}$ such that $\sigma \rho=(12 \cdots n)$ where, of course, $(12 \cdots n) \in \mathscr{C}_{(n)}$.

Dénes [6] (see also Dénes [7] and Hurwitz [11, 12]) had previously shown that

$$
c_{\alpha_{1}, \ldots, \alpha_{n-1}}^{(n)}=n^{n-2}
$$

where $\alpha_{1}, \ldots, \alpha_{n-1}$ are transpositions (i.e., $\alpha_{i}=1^{n-2} 2$ for $i=1, \ldots, n-1$ ). In Moszkowski [18] an explicit bijection is given between labelled trees on $n$ vertices (it is well known (see, e.g., Cayley [5]) that the number of these is $n^{n-2}$ ) and $(n-1)$-tuples of transpositions whose product is $(12 \cdots n)$.

One of the results (Corollary 4.2) in Jackson [15] specializes to give the following result.

Theorem 1.1. For $\alpha_{i}=1^{n-2} 2, i=1, \ldots, m$ and $m \geq 1$,

$$
c_{\alpha_{1}, \ldots, \alpha_{m}}^{(n)}=\frac{1}{n !} \sum_{k=0}^{n-1}\left\{\left(\begin{array}{l}
n \\
2
\end{array}\right)-n k\right\}^{m}\left(\begin{array}{c}
n-1 \\
k
\end{array}\right)(-1)^{k} .
$$

Note that when $m=n-1$ the RHS of Theorem 1.1 must reduce to $n^{n-2}$ from (1.2), and that when $m<n-1$ the RHS must reduce to 0 , for combinatorial reasons.

The purpose of this paper is to derive an explicit bijective proof of Theorem 1.1. We proceed by considering a generalization of Theorem 1.1 that allows us to identify the role of the summation index $k$. This generalization is given as Theorem 2.1, and is referred to as the main result.

There are three stages. First, in $\S 2$, we derive the main result in a straightforward manner from the character theory of $S_{n}$. Second, in $\S 3$, we give a noncharacter, but essentially verificational, proof of the main result by means of a partial differential operator for symmetric functions. Third, in $\S 4$, we are able to derive an explicit bijective proof of the main result by considering the combinatorial implications of the action of this differential operator.

The common thread running through these three stages is the formal power series

$$
A(r, b, t ; \mathbf{x})=(r-b)^{-1}\{H(r t) E(-b t)-1\}
$$


where

$$
H(u)=\prod_{j \geq 1}\left(1-u x_{j}\right)^{-1}, \quad E(u)=\prod_{j \geq 1}\left(1+u x_{j}\right)
$$

are well-known as the generating functions for the complete and elementary symmetric functions in $\mathbf{x}=\left(x_{1}, x_{2}, \ldots\right)$, respectively.

The reader is referred to Macdonald [17] for a complete treatment of symmetric functions. In this paper we shall be concerned only with the power sum symmetric functions, given by

$$
p_{0}=1, \quad p_{j}=x_{1}^{j}+x_{2}^{j}+\ldots, \quad j \geq 1 .
$$

If $\lambda=\left(\lambda_{1}, \lambda_{2}, \ldots\right)$ is a partition, then $p_{\lambda}=p_{\lambda_{1}} p_{\lambda_{2}} \ldots$ and $\left\{p_{\lambda} \mid \lambda\right.$ all partitions $\}$ forms a basis for symmetric functions, where $p_{1}, p_{2}, \ldots$ are algebraically independent. The generating function for $p_{1}, p_{2}, \ldots$ is denoted by $P(u)=\sum_{j \geq 1} p_{j} u^{j}$. The $p_{j}$ 's are related to $H$ and $E$ by

$$
H(u)=\exp \left(\sum_{j \geq 1} p_{j} \frac{u^{j}}{j}\right), \quad E(-u)=\exp \left(-\sum_{j \geq 1} p_{j} \frac{u^{j}}{j}\right)
$$

and thus

$$
u \frac{\partial}{\partial u} H(u)=P(u) H(u), \quad u \frac{\partial}{\partial u} E(-u)=-P(u) E(-u)
$$

Various properties of the series $A$ that we shall need are given in the following result, in which $[M] N$ denotes "the coefficient of $M$ in $N$ ".

\section{Proposition 1.2.}

(1)

$$
A(r, b, t ; \mathbf{x})=\sum_{n \geq 1} \sum_{k=0}^{n-1} \frac{t^{n}}{n !} r^{n-1-k} b^{k} A_{n, k}
$$

$$
A_{n, k}=\sum_{\alpha \vdash n} a_{n, k, \alpha}\left|\mathscr{C}_{\alpha}\right| p_{\alpha}, \quad \text { where }\left|\mathscr{C}_{\alpha}\right|=n ! / \prod_{j \geq 1} j^{i_{j}} i_{j} ! \text { if } \alpha=1^{i_{1}} 2^{i_{2}} \ldots
$$

$$
a_{n, k, \alpha}=\left[r^{n-1-k} b^{k}\right](r-b)^{-1} \prod_{j \geq 1}\left(r^{j}-b^{j}\right)^{i_{j}} \quad \text { where } \alpha=1^{i_{1}} 2^{i_{2}} \ldots,
$$

(4) $A(1,1, t ; \mathbf{x})=P(t)$,

(5) $\sum_{k=0}^{n-1} a_{n, k, \alpha}=n \delta_{\alpha,(n)}$ for $\alpha \vdash n$.

Proof. (1) The division by $r-b$ in the definition of $A$ is well-defined when $A$ is considered as a power series in $t$. As a polynomial in $r, b$, the coefficient of $t^{n}$ in $H(r t) E(-b t)-1$ is homogeneous of degree $n$, for $n \geq 1$ (and is 0 for $n=0$ since $H(0)=E(0)=1)$. Moreover, $H(r t) E(-b t)-1=0$ when $r=b$, so its coefficient of $t^{n}$ is divisible by $r-b$. Thus, as a polynomial in $r, b$, the coefficient of $t^{n}$ in $A$ is homogeneous of degree $n-1, n \geq 1$. Accordingly, we can write $A$ in the given form, where the division by $n$ ! is for convenience later. 
(2) $A$ is a function of $t$ and $x_{j}$ only through $t x_{j}, j \geq 1$, and $A$ is a symmetric function in $\mathbf{x}$, so the $A_{n, k}$ are symmetric functions in $\mathbf{x}$ of degree $n$. The result follows because $\left\{p_{\alpha} \mid \alpha \vdash n\right\}$ is a basis for symmetric functions of degree $n$. Again the multiplication by $\left|\mathscr{C}_{\alpha}\right|$ is for convenience later, and the value of $\left|\mathscr{C}_{\alpha}\right|$ is well-known.

(3) From (1.3) we have

$$
\begin{aligned}
A & =(r-b)^{-1}\left\{\exp \left(\sum_{j \geq 1} p_{j} \frac{t^{j}}{j}\left(r^{j}-b^{j}\right)\right)-1\right\} \\
& =(r-b)^{-1}\left\{\prod_{j \geq 1} \exp \left(p_{j} \frac{t_{j}}{j}\left(r^{j}-b^{j}\right)\right)-1\right\} \\
& =(r-b)^{-1} \sum_{n \geq 1} \frac{t^{n}}{n !} \sum_{\alpha=1^{i_{1} 2^{i_{2}} \ldots+n}}\left|\mathscr{C}_{\alpha}\right| p_{\alpha} \prod_{j \geq 1}\left(r^{j}-b^{j}\right)^{i_{j}} .
\end{aligned}
$$

This gives immediately, from (2) and (1),

$$
a_{n, k, \alpha}=\left[\frac{t^{n}}{n !} r^{n-1-k} b^{k}\left|\mathscr{C}_{\alpha}\right| p_{\alpha}\right] A=\left[r^{n-1-k} b^{k}\right](r-b)^{-1} \prod_{j \geq 1}\left(r^{j}-b^{j}\right)^{i_{j}}
$$

as required.

(4) Using L'Hôpital's rule, which is justified since $r$ and $b$ appear polynomially as coefficients of powers of $t$,

$$
\begin{aligned}
A(1,1, t ; \mathbf{x}) & =\lim _{r \rightarrow 1} A(r, 1, t ; \mathbf{x})=\lim _{r \rightarrow 1} \frac{H(r t) E(-t)-1}{r-1} \\
& =\left.\frac{\partial}{\partial r}\{H(r t) E(-t)-1\}\right|_{r=1}=P(t) H(t) E(-t),
\end{aligned}
$$

and the result follows since $H(t) E(-t)=1$.

(5) From (1) and (2) we have

$$
A(1,1, t ; \mathbf{x})=\sum_{n \geq 1} \sum_{k=0}^{n-1} \frac{t^{n}}{n !} \sum_{\alpha \vdash n} a_{n, k, \alpha}\left|\mathscr{C}_{\alpha}\right| p_{\alpha},
$$

so that

$$
\begin{aligned}
& \sum_{k=0}^{n-1} a_{n, k, \alpha}=\left[\frac{t^{n}}{n !}\left|\mathscr{C}_{\alpha}\right| p_{\alpha}\right] A(1,1, t ; \mathbf{x}) \\
& =\left[\frac{t^{n}}{n !}\left|\mathscr{C}_{\alpha}\right| p_{\alpha}\right] P(t) \quad \text { from (4), } \\
& = \begin{cases}n ! /\left|\mathscr{C}_{(n)}\right|, & \alpha=(n), \\
0, & \text { otherwise. }\end{cases}
\end{aligned}
$$

But $\left|\mathscr{C}_{(n)}\right|=n ! / n$ from (2) and the result follows.

The main result is stated in terms of the integers $a_{n, k, \alpha}$. In $\S 2$ these integers are identified in terms of characters of $S_{n}$, and properties of characters are used in deriving the main result there. In $\S 3$ a differential operator for symmetric functions is introduced, and its action on $A$ is shown to be nice; the main result 
follows, with the $a_{n, k, \alpha}$ appearing only as the coefficients in the symmetric function $A$. In $\S 4$ we demonstrate that the series $A$ is the generating function with respect to an alternating weight for a set of combinatorial objects that we call properly painted permutations. This leads to a combinatorial derivation of the action of the differential operator on this generating function. In $\$ 5$ we extend this to obtain a direct bijective proof of the main result, in which the $a_{n, k, \alpha}$ appear as the combinatorial sum of alternating weights for sets of painted permutations. This quickly leads to a direct bijection for Theorem 1.1 (with both sides multiplied by $2^{m} n$ !).

\section{Characters OF THE SYMMETRIC GROUP}

It is well-known (see, e.g., Burrow [4]) that the centre of the group algebra $\mathbb{C} S_{n}$ has a basis $\left\{F_{\alpha} \mid \alpha \vdash n\right\}$ of orthogonal idempotents, given by

$$
F_{\alpha}=\frac{f^{\alpha}}{n !} \sum_{\beta \vdash n} \chi_{\beta}^{\alpha} K_{\beta},
$$

where $\chi_{\beta}^{\alpha}$ is the character of the irreducible representation of $S_{n}$ associated with $\mathscr{C}_{\alpha}$, evaluated at $\mathscr{C}_{\beta}$, and $f^{\alpha}$ is the degree of this representation. Moreover we can invert the linear relationship (2.1) to obtain

$$
K_{\alpha}=\left|\mathscr{C}_{\alpha}\right| \sum_{\beta \vdash n} \frac{1}{f^{\beta}} \chi_{\alpha}^{\beta} F_{\beta} .
$$

The introduction of characters of $S_{n}$ is relevant because the series $A_{n, k}$ discussed in $\S 1$ is essentially the generating function for the values of the character of the irreducible representation associated with $C_{\left(1^{k}, n-k\right)}$. In particular, as derived on page 139 of Littlewood [16],

$$
a_{n, k, \alpha}=(-1)^{k} \chi_{\alpha}^{\left(1^{k}, n-k\right)} \text {. }
$$

We are now ready to give the main result, which yields a value for a linear combination of connection coefficients involving arbitrary powers of transpositions. The proof exploits the above close connection between $a_{n, k, \alpha}$ and characters.

Theorem 2.1. For $n \geq 1, n>k \geq 0, \alpha_{i}=1^{n-2} 2$ for $i=1, \ldots, m, m \geq 0$, and $\beta \vdash n$,

$$
\sum_{\alpha \vdash n} a_{n, k, \alpha} c_{\alpha_{1}, \ldots, \alpha_{m}, \alpha}^{\beta}=\left\{\left(\begin{array}{l}
n \\
2
\end{array}\right)-n k\right\}^{m} a_{n, k, \beta} .
$$

Proof. Since $a_{n, k, \alpha}=(-1)^{k} \chi_{\alpha}^{\left(1^{k}, n-k\right)}$, we have

$$
(-1)^{k} \frac{f^{\left(1^{k}, n-k\right)}}{n !} \sum_{\alpha \vdash n} a_{n, k, \alpha} K_{\alpha}=F_{\left(1^{k}, n-k\right)},
$$

from (2.1). Thus from (2.1), (2.2),

$$
\left\{K_{\left(1^{n-2} 2\right)}\right\}^{m} \sum_{\alpha \vdash n} a_{n, k, \alpha} K_{\alpha}=\left\{\frac{\left|\mathscr{C}_{\left(1^{n-2} 2\right)}\right| \chi_{\left(1^{n-2} 2\right)}^{\left(1^{k}, n-k\right)}}{f^{\left(1^{k}, n-k\right)}}\right\}^{m} \frac{(-1)^{k} n ! F_{\left(1^{k}, n-k\right)}}{f^{\left(1^{k}, n-k\right)}} .
$$


But

$$
\begin{aligned}
f^{\left(1^{k}, n-k\right)} & =\chi_{\left(1^{n}\right)}^{\left(1^{k}, n-k\right)} \\
& =(-1)^{k} a_{n, k,\left(1^{n}\right)}, \quad \text { from }(2.3) \\
& =(-1)^{k}\left[r^{n-1-k} b^{k}\right](r-b)^{n-1}, \quad \text { from Proposition 1.2(3) } \\
& =\left(\begin{array}{c}
n-1 \\
k
\end{array}\right), \\
\chi_{\left(1^{n-22}\right)}^{\left(1^{k}, n-k\right)} & =(-1)^{k} a_{n, k,\left(1^{n-2}\right)}, \quad \text { from }(2.3) \\
& =(-1)^{k}\left[r^{n-1-k} b^{k}\right](r-b)^{n-2}(r+b), \quad \text { from Proposition 1.2(3) } \\
& =\left(\begin{array}{c}
n-2 \\
k
\end{array}\right)-\left(\begin{array}{c}
n-2 \\
k-1
\end{array}\right)
\end{aligned}
$$

and

$$
\left|\mathscr{C}_{\left(1^{n-2} 2\right)}\right|=\frac{n !}{(n-2) ! 2}=\left(\begin{array}{l}
n \\
2
\end{array}\right), \quad \text { from Proposition 1.2(2) }
$$

Thus

$$
\frac{\left|\mathscr{C}_{\left(1^{n-2} 2\right)}\right| \chi_{\left(1^{n-2}\right)}^{\left(1^{k}, n-k\right)}}{f^{\left(1^{k}, n-k\right)}}=\frac{\left(\begin{array}{c}
n \\
2
\end{array}\right)\left\{\left(\begin{array}{c}
n-2 \\
k
\end{array}\right)-\left(\begin{array}{c}
n-2 \\
k-1
\end{array}\right)\right\}}{\left(\begin{array}{c}
n-1 \\
k
\end{array}\right)}=\left(\begin{array}{l}
n \\
2
\end{array}\right)-n k,
$$

and substitution of this in (2.5) gives

$$
\begin{aligned}
\left\{K_{\left(1^{n-2} 2\right)}\right\}^{m} \sum_{\alpha \vdash n} a_{n, k, \alpha} K_{\alpha} & =\left\{\left(\begin{array}{l}
n \\
2
\end{array}\right)-n k\right\}^{m} \frac{(-1)^{k} n !}{\left(\begin{array}{c}
n-1 \\
k
\end{array}\right)} F_{\left(1^{k}, n-k\right)} \\
& =\left\{\left(\begin{array}{l}
n \\
2
\end{array}\right)-n k\right\}^{m} \sum_{\beta \vdash n} a_{n, k, \beta} K_{\beta}, \quad \text { from }(2.1),(2.4) .
\end{aligned}
$$

The result follows immediately by equating coefficients of $K_{\beta}$ on both sides of this expression.

The above result is closely related to those of Jackson [15]. From it we deduce the value of a connection coefficient involving arbitrary powers of transpositions in the next result.

Corollary 2.2. For $n \geq 1, \alpha_{i}=1^{n-2} 2$ for $i=1, \ldots, m, m \geq 0$, and $\beta \vdash n$,

$$
c_{\alpha_{1}, \ldots, \alpha_{m},(n)}^{\beta}=\frac{1}{n} \sum_{k=0}^{n-1}\left\{\left(\begin{array}{l}
n \\
2
\end{array}\right)-n k\right\}^{m} a_{n, k, \beta} .
$$

Proof. Summing both sides of Theorem 2.1 over $k=0, \ldots, n-1$ and equating the results yields

$$
\sum_{\alpha \vdash n}\left(\sum_{k=0}^{n-1} a_{n, k, \alpha}\right) c_{\alpha_{1}, \ldots, \alpha_{m}, \alpha}^{\beta}=\sum_{k=0}^{n-1}\left\{\left(\begin{array}{l}
n \\
2
\end{array}\right)-n k\right\}^{m} a_{n, k, \beta} .
$$

But

$$
\sum_{\alpha \vdash n}\left(\sum_{k=0}^{n-1} a_{n, k, \alpha}\right) c_{\alpha_{1}, \ldots, \alpha_{m}, \alpha}^{\beta}=n c_{\alpha_{1}, \ldots, \alpha_{m},(n)}^{\beta}
$$

from Proposition 1.2(5), and the result follows upon division by $n$. 
We obtain Theorem 1.1 in a straightforward way by considering the special case $\beta=\left(1^{n}\right)$ of Corollary 2.2.

Proof of Theorem 1.1. From Corollary 2.2, the number of $m$-tuples of transpositions $\left(\tau_{1} \cdots \tau_{m}\right)$ and $n$-cycles $\sigma$ such that $\tau_{1} \cdots \tau_{m} \sigma=\mathrm{id}$ is given by

$$
c_{\alpha_{1}, \ldots, \alpha_{m},(n)}^{\left(1^{n}\right)}=\frac{1}{n} \sum_{k=0}^{n-1}\left\{\left(\begin{array}{l}
n \\
2
\end{array}\right)-n k\right\}^{m} a_{n, k,\left(1^{n}\right)},
$$

where $\alpha_{i}=1^{n-2} 2$ for $i=1, \ldots, m$. But $a_{n, k,\left(1^{n}\right)}=(-1)^{k}\left(\begin{array}{c}n-1 \\ k\end{array}\right)$, as derived in the proof of Theorem 2.1.

Furthermore $\tau_{1} \cdots \tau_{m} \sigma=\mathrm{id}$ is equivalent to $\tau_{1} \cdots \tau_{m}=\sigma^{-1}$, where $\sigma^{-1}$ is also an $n$-cycle, so

The result follows since $\left|\mathscr{C}_{(n)}\right|=(n-1)$ !

$$
c_{\alpha_{1}, \ldots, \alpha_{m},(n)}^{\left(1^{n}\right)}=\left|\mathscr{C}_{(n)}\right| \cdot c_{\alpha_{1}, \ldots, \alpha_{m}}^{(n)} .
$$

\section{A DIFFERENTIAL OPERATOR FOR SYMMETRIC FUNCTIONS}

For $\sigma \in S_{n}$, let $\Phi(\sigma)=p_{d(\sigma)}$, and linearly extend this to define the action of $\Phi$ on $\mathbb{C} S_{n}$. Thus for example, $\Phi\left(K_{\alpha}\right)=\left|\mathscr{C}_{\alpha}\right| p_{\alpha}$ and $\Phi\left(\sum_{\alpha \vdash n} K_{\alpha}\right)=h_{n}$.

Now define the differential operator for symmetric functions (it is welldefined since the power sum symmetric functions are algebraically independent).

$$
\Delta=\frac{1}{2} \sum_{i \geq 1} \sum_{j \geq 1}\left(i j p_{i+j} \frac{\partial}{\partial p_{i}} \frac{\partial}{\partial p_{j}}+(i+j) p_{i} p_{j} \frac{\partial}{\partial p_{i+j}}\right) .
$$

This operator provides a realization in the algebra of symmetric functions of the multiplication by all transpositions in the symmetric group, as demonstrated in the next result.

Proposition 3.1. For $g \in \mathbb{C} S_{n}$,

$$
\Phi\left(K_{\left(1^{n-2}\right)} g\right)=\Delta \Phi(g) .
$$

Proof. Consider an arbitrary transposition $\left(t_{1}, t_{2}\right)$ and an arbitrary permutation $\sigma \in S_{n}$.

If $t_{1}$ and $t_{2}$ appear on two different cycles in the disjoint cycle representation of $\sigma$, then in the product $\left(t_{1}, t_{2}\right) \sigma$ those cycles are replaced by a single cycle whose length is equal to the sum of their lengths. Thus a $p_{i} p_{j}$ in $\Phi(\sigma)$ is replaced by a $p_{i+j}$ in $\Phi\left(\left(t_{1}, t_{2}\right) \sigma\right)$ for some $i, j \geq 1$.

If $t_{1}$ and $t_{2}$ appear on the same cycle in $\sigma$, then in the product $\left(t_{1}, t_{2}\right) \sigma$ that cycle is replaced by two cycles whose lengths sum to the length of that cycle. Thus a $p_{i+j}$ in $\Phi(\sigma)$ is replaced by a $p_{i} p_{j}$ in $\Phi\left(\left(t_{1}, t_{2}\right) \sigma\right)$ for some $i, j \geq 1$.

The result follows by considering all cases for $\left(t_{1}, t_{2}\right)$ and extending linearly to $\mathbb{C} S_{n}$.

As an exercise, the reader might verify that

$$
\Delta H(t)=\frac{1}{2} \frac{\partial^{2}}{\partial t^{2}} H(t), \quad \Delta E(t)=-\frac{1}{2} \frac{\partial^{2}}{\partial t^{2}} E(t) .
$$

This operator also acts very nicely on the series $A$ discussed in $\S 1$, as described in the following result. 


\section{Theorem 3.2.}

(1)

$$
\Delta A=\frac{1}{2}\left(r \frac{\partial}{\partial r}-b \frac{\partial}{\partial b}\right) t \frac{\partial}{\partial t} A
$$

$$
\Delta A_{n, k}=\left\{\left(\begin{array}{l}
n \\
2
\end{array}\right)-n k\right\} A_{n, k}, \quad \text { for } n>k \geq 0 .
$$

Proof. (1)

$$
\begin{aligned}
& \Delta A=(r-b)^{-1} \Delta\left\{\exp \left(\sum_{k \geq 1} p_{k} \frac{t^{k}}{k}\left(r^{k}-b^{k}\right)\right)-1\right\}, \quad \text { from }(1.3) \\
&=\frac{H(r t) E(-b t)}{2(r-b)} \sum_{i, j \geq 1}\left\{p_{i+j} t^{i+j}\left(r^{i}-b^{i}\right)\left(r^{j}-b^{j}\right)+p_{i} p_{j} t^{i+j}\left(r^{i+j}-b^{i+j}\right)\right\} \\
&=\frac{H(r t) E(-b t)}{2(r-b)}\left\{\sum_{m \geq 1} p_{m} t^{m}\left(m r^{m}+m b^{m}-\sum_{i=0}^{m-1}\left(r^{i} b^{m-i}+r^{m-i} b^{i}\right)\right)\right. \\
&\left.+P(r t)^{2}-P(b t)^{2}\right\} \\
&=\frac{H(r t) E(-b t)}{2(r-b)}\left\{r t P^{\prime}(r t)+b t P^{\prime}(b t)-\frac{r+b}{r-b}(P(r t)-P(b t))\right. \\
&\left.+P(r t)^{2}-P(b t)^{2}\right\} .
\end{aligned}
$$

But

so

$$
t \frac{\partial}{\partial t} A=\frac{H(r t) E(-b t)}{r-b}\{P(r t)-P(b t)\},
$$

$$
\begin{aligned}
& r \frac{\partial}{\partial r} t \frac{\partial}{\partial t} A=\frac{H(r t) E(-b t)}{r-b}\left\{r t P^{\prime}(r t)+\left(P(r t)-\frac{r}{r-b}\right)(P(r t)-P(b t))\right\} \\
& b \frac{\partial}{\partial b} t \frac{\partial}{\partial t} A=\frac{H(r t) E(-b t)}{r-b}\left\{-b t P^{\prime}(b t)-\left(P(b t)-\frac{b}{r-b}\right)(P(r t)-P(b t))\right\}
\end{aligned}
$$

and the result follows.

(2) We have

so

$$
A=\sum_{n>k \geq 0} A_{n, k} r^{n-1-k} b^{k} \frac{t^{n}}{n !}
$$

and

$$
\Delta A=\sum_{n>k \geq 0}\left(\Delta A_{n, k}\right) r^{n-1-k} b^{k} \frac{t^{n}}{n !}
$$

$$
\begin{aligned}
\frac{1}{2}\left(r \frac{\partial}{\partial r}-b \frac{\partial}{\partial b}\right) t \frac{\partial}{\partial t} A & =\sum_{n>k \geq 0} A_{n, k}\left\{\frac{1}{2}\left(r \frac{\partial}{\partial r}-b \frac{\partial}{\partial b}\right) t \frac{\partial}{\partial t} r^{n-1-k} b^{k} \frac{t^{n}}{n !}\right\} \\
& =\sum_{n>k \geq 0} A_{n, k}\left\{\frac{1}{2}(n-1-k-k) n\right\} r^{n-1-k} b^{k} \frac{t^{n}}{n !}
\end{aligned}
$$


Apply (1) and equate coefficients of $r^{n-1-k} b^{k} t^{n} / n$ ! on both sides to obtain

$$
\Delta A_{n, k}=\left(\frac{1}{2}(n-1-2 k) n\right) A_{n, k},
$$

for $n>k \geq 0$, and the result follows.

The above properties of $\Delta$ combine to yield the following proof of the main result.

Second proof of Theorem 2.1. From Proposition 3.1

$$
\begin{aligned}
\Phi\left(K_{\left(1^{n-22)}\right.}^{m} \sum_{\alpha \vdash n} a_{n, k, \alpha} K_{\alpha}\right) & =\Delta^{m} \Phi\left(\sum_{\alpha \vdash n} a_{n, k, \alpha} K_{\alpha}\right)=\Delta^{m} \sum_{\alpha \vdash n} a_{n, k, \alpha} \Phi\left(K_{\alpha}\right) \\
& =\Delta^{m} \sum_{\alpha \vdash n} a_{n, k, \alpha}\left|\mathscr{C}_{\alpha}\right| p_{\alpha}=\Delta^{m}\left(A_{n, k}\right) \\
& =\left\{\left(\begin{array}{l}
n \\
2
\end{array}\right)-n k\right\}^{m} A_{n, k}, \quad \text { from Theorem 3.2(2) } \\
& =\Phi\left(\left\{\left(\begin{array}{l}
n \\
2
\end{array}\right)-n k\right\}^{m} \sum_{\beta \vdash n} a_{n, k, \beta} K_{\beta}\right)
\end{aligned}
$$

and the result follows.

Thus we have obtained a proof of the results of $\S 2$ that is free of the use of characters. By this we refer to the fact that no use is made of the relationship between the $a_{n, k, \alpha}$ and characters; instead the $a_{n, k, \alpha}$ are regarded simply as coefficients in the symmetric function $A$, and the action of $\Delta$ on $A$ is then explicitly described.

This proof, though free of characters, is essentially verificational, since it is based on the fact that $A$ satisfies the partial differential equation given in Theorem 3.2(1). However, we are able to derive a constructive, directly combinatorial, proof of Theorem 3.2 in the next section, which leads to a combinatorial proof of the main result in $\S 5$.

\section{Painted permutations AND the differential operator}

By a painted permutation $\rho$ on $\{1, \ldots, n\}$ we shall mean a permutation $\sigma \in S_{n}$ in which each element $1, \ldots, n-1$ is assigned ("painted") one of the colours red or blue, and we say in this situation that $\rho$ is obtained by painting $\sigma$. Thus there are $2^{n-1}$ ways to paint $\sigma$ for each $\sigma \in S_{n}$, giving a total of $2^{n-1} n$ ! painted permutations on $\{1, \ldots, n\}$. We shall be concerned in this section with a special subset of painted permutations called properly painted permutations, defined below.

In the disjoint cycle representation of a permutation $\sigma \in S_{n}$, suppose that the cycle containing element $n$ is $\left(n, i_{1}, i_{2}, \ldots, i_{j}\right)$, and call this cycle the maxcycle of $\sigma$. Consider painting elements $i_{1}, \ldots, i_{l}$ red and elements $i_{l+1}, \ldots, i_{j}$ blue for some $0 \leq l \leq j$, and denote the resulting object by $\left(n,\left[i_{1}, \ldots, i_{l}\right]_{R}\right.$, $\left[i_{l+1}, \ldots, i_{j}\right]_{B}$ ) (if $l=0$ then []$_{R}$ is suppressed and if $l=j$ then []$_{B}$ is suppressed). For every other cycle $\left(m_{1}, \ldots, m_{u}\right)$ in $\sigma$ either paint all elements $m_{1}, \ldots, m_{u}$ red or all blue, and denote the resulting object $\left(m_{1}, \ldots, m_{u}\right)_{R}$ or $\left(m_{1}, \ldots, m_{u}\right)_{B}$, respectively. The result of this procedure is a properly 
painted permutation $\rho$, and we say that $\rho$ is obtained by (properly) painting $\sigma$. Defined $B(\rho)$ to be the number of cycles of $\rho$ in which all elements are blue, and define $\Phi(\rho)=\Phi(\sigma)$, where $\Phi(\sigma)$ is given in $\S 3$, and $\rho$ is obtained by painting $\sigma$.

Let the set of properly painted permutations on $n$ elements of which $k$ are blue (and hence $n-1-k$ are red) be denoted by $\mathscr{A}_{n, k}$. For $\rho \in \mathscr{A}_{n, k}$, define the weight function

For example, with $n=9, k=3$,

$$
w t(\rho)=(-1)^{B(\rho)} \Phi(\rho) .
$$

$$
w t\left(\left(9,[6,3]_{R},[5]_{B}\right)(1,8)_{B}(2,7,4)_{R}\right)=-p_{2} p_{3} p_{4} .
$$

The significance of properly painted permutations is that the generating function for $\mathscr{A}_{n, k}$ with respect to this weight function is the series $A_{n, k}$ discussed in $\S 1$, as proved in the next result.

Proposition 4.1. For $n>k \geq 0$,

Proof. Let

$$
\sum_{\rho \in \mathscr{A}_{n, k}} w t(\rho)=A_{n, k}
$$

$$
D_{n}=\sum_{k=0}^{n-1} r^{n-1-k} b^{k} \sum_{\rho \in \mathscr{A}_{n, k}} w t(\rho)=\sum_{\sigma \in S_{n}} p_{d(\sigma)} G_{\sigma}
$$

Then

$$
G_{\sigma}=\sum_{\rho}(-1)^{B(\rho)} r^{\# \text { red elements in } \rho} b^{\# \text { blue elements in } \rho}
$$

where the summation is over all $\rho$ obtained by properly painting $\sigma$.

Now suppose $\sigma \in \mathscr{C}_{\left(1_{1} 2^{i_{2} \ldots}\right)}$, so $\sigma$ has $i_{j}$ cycles of length $j$, and that the max-cycle has length $m$. Then

$$
G_{\sigma}=\left(\sum_{l=0}^{m-1} r^{l} b^{m-1-l}\right) \prod_{j \geq 1}\left(r^{j}-b^{j}\right)^{i_{j}-\delta_{j, m}}
$$

since the max-cycle has $l$ red elements and $m-1-l$ blue elements for some $l=0, \ldots, m-1$, and each other cycle has, independently, all elements red or blue, with the number of the latter giving $B(\rho)$. But

$$
\sum_{l=0}^{m-1} r^{l} b^{m-1-l}=(r-b)^{-1}\left(r^{m}-b^{m}\right)
$$

so

$$
G_{\sigma}=(r-b)^{-1} \prod_{j \geq 1}\left(r^{j}-b^{j}\right)^{i_{j}} .
$$

Thus $G_{\sigma}$ depends only on the conjugacy class of $\sigma$, so

$$
D_{n}=(r-b)^{-1} \sum_{\alpha=1^{i_{1}} 2^{i_{2} \ldots+n}}\left|\mathscr{C}_{c}\right| p_{\alpha} \prod_{j \geq 1}\left(r^{j}-b^{j}\right)^{i_{j}} .
$$

Comparing this expression with Proposition 1.2, we immediately have

$$
\left[r^{k} b^{n-1-k}\right] D_{n}=A_{n, k},
$$

as required. 
For $\tau \in S_{n}$ and $\rho$ a painted permutation, we define $\tau \rho$ (or $\rho \tau$ ) as follows. If $\rho$ is obtained by painting $\sigma$ then $\tau \rho$ (or $\rho \tau$ ) is the permutation $\tau \sigma$ (or $\sigma \tau)$ with elements the same colour as in $\rho$. Note that either or both of $\rho$ and $\tau \rho$ (or $\rho \tau$ ) may be properly painted.

Now define sets $\mathscr{S}_{n, k}$ and $\mathscr{T}_{n, k}$ by

$$
\begin{gathered}
\mathscr{S}_{n, k}=\left\{(u, v, \rho) \mid 1 \leq u \neq v \leq n, \rho \in \mathscr{A}_{n, k}\right\}, \\
\mathscr{T}_{n, k}=\left\{(y, z, \gamma) \mid 1 \leq y \leq n-1,1 \leq z \leq n, \gamma \in \mathscr{A}_{n, k}\right\} .
\end{gathered}
$$

For $(u, v, \rho) \in \mathscr{S}_{n, k}$, define the weight function

$$
\xi(u, v, \rho)=(-1)^{B(\rho)} p_{d((u, v) \rho)},
$$

and for $(y, z, \gamma) \in \mathscr{T}_{n, k}$, define

$$
\omega(y, z, \rho)= \begin{cases}\omega t(\gamma) & \text { if element } y \text { is red in } \gamma, \\ -\omega t(\gamma) & \text { if element } y \text { is blue in } \gamma .\end{cases}
$$

Generating functions with respect to these weight functions are given by

$$
\begin{array}{ll}
\Omega_{\mathscr{S}}(\mathbf{x})=\sum_{(u, v, \rho) \in \mathscr{S}} \xi(u, v, \rho) & \text { for } \mathscr{S} \subseteq \mathscr{I}_{n, k}, \\
\Psi_{\mathscr{T}}(\mathbf{x})=\sum_{(y, z, \gamma) \in \mathscr{T}} \omega(y, z, \gamma) & \text { for } \mathscr{T} \subseteq \mathscr{T}_{n, k} .
\end{array}
$$

The connection to $\S 3$ is made clear in the following result.

Proposition 4.2. For $n>k \geq 0$,

(1) $\Omega_{\mathscr{S}_{n, k}}(\mathbf{x})=2 \Delta A_{n, k}$,

(2) $\Psi_{\mathscr{T}_{n, k}}(\mathbf{x})=2\left\{\left(\begin{array}{l}n \\ 2\end{array}\right)-n k\right\} A_{n, k}$.

Proof. (1) The result follows immediately from Propositions 3.1 and 4.1.

(2) The result follows from Proposition 4.1 since for each $\gamma \in \mathscr{A}_{n, k}$, there are $n$ choices for $z$ and $n-1$ choices for $y$. There are $n-1-k$ choices for $y$ specifying red elements and $k$ specifying blue elements. The latter reverse the sign, so

$$
\Psi_{\mathscr{S}_{n, k}}(\mathbf{x})=\{(n-1-k)-k\} n A_{n, k} .
$$

Note that Theorem 3.2 and Proposition 4.2 imply

$$
\Omega_{\mathscr{S}_{n, k}}(\mathbf{x})=\Psi_{\mathscr{T}_{n, k}}(\mathbf{x}) .
$$

This means that the effect of multiplying all properly painted permutations in $\mathscr{A}_{n, k}$ by the set of transpositions $\left(u, v\right.$ is the transposition in $\left.\mathscr{S}_{n, k}\right)$ is equivalent to "marking" all pairs of (not necessarily distinct) elements in all properly painted permutations in $\mathscr{A}_{n, k}$, where the first marked element is painted (i.e., not $n$ ) and a sign of -1 is introduced if this element is blue $(y, z$ are the marked elements in $\mathscr{T}_{n, k}$ ). For a general discussion of the relationship between the action of $t \partial / \partial t$ (and $r \partial / \partial r, b \partial / \partial b$ ) as on the RHS of Theorem 3.2.(1) and marking (or rooting or distinguishing) of combinatorial objects see, e.g., Goulden and Jackson [8].

In the remainder of this section we complete a purely combinatorial proof of Theorem 3.2 by deriving a direct combinatorial proof of $(4.1)$, which together 
with Proposition 4.2 implies Theorem 3.2 (multiplied by 2). This proof of (4.1) proceeds in the following way. First, we partition $\mathscr{S}_{n, k}$ into subsets $\mathscr{S}_{n, k}^{(1)}$ and $\mathscr{S}_{n, k}^{(2)}$ and give a sign-reversing involution $\eta$ on $\mathscr{S}_{n, k}^{(1)}$, proving that $\boldsymbol{\Omega}_{\mathscr{S}_{n, k}^{(1)}}(\mathbf{x})=0$. Second we partition $\mathscr{T}_{n, k}$ into subsets $\mathscr{T}_{n, k}^{(1)}$ and $\mathscr{T}_{n, k}^{(2)}$ and give a sign-reversing involution $\psi$ on $\mathscr{T}_{n, k}^{(1)}$, proving that $\Psi_{\mathscr{T}_{n, k}^{(1)}}(\mathbf{x})=0$. Finally, we give a weight-preserving bijection $\phi$ between $\mathscr{S}_{n, k}^{(2)}$ and $\mathscr{T}_{n, k}^{(2)}$, proving that $\Omega_{\mathscr{S}_{n, k}^{(2)}}=\Psi_{\mathscr{G}_{n, k}^{(2)}}$.

The sets $\mathscr{S}_{n, k}^{(1)}$ and $\mathscr{T}_{n, k}^{(1)}$ are given by

$$
\begin{aligned}
& \mathscr{S}_{n, k}^{(1)}=\left\{(u, v, \rho) \in \mathscr{S}_{n, k} \mid \text { elements } u, v \text { have different colours in } \rho\right\}, \\
& \mathscr{T}_{n, k}^{(1)}=\left\{(y, z, \gamma) \in \mathscr{T}_{n, k} \mid \text { elements } y, z \text { have different colours in } \gamma\right\},
\end{aligned}
$$

where "different colours" means, in both cases, that one is red and the other blue. The sets $\mathscr{S}_{n, k}^{(2)}$ and $\mathscr{T}_{n, k}^{(2)}$ are their complements in $\mathscr{S}_{n, k}$ and $\mathscr{T}_{n, k}$.

The easiest of the mappings is $\psi$; for $(y, z, \gamma) \in \mathscr{T}_{n, k}^{(1)}$, define

$$
\psi(y, z, \gamma)=(z, y, \gamma) \text {. }
$$

(Note that $z \neq n$ since $z$ is painted.)

Theorem 4.3. For $n>k \geq 0$,

(1) $\psi$ is a fixed-point-free involution on $\mathscr{T}_{n, k}^{(1)}$,

(2) $\omega(\psi(y, z, \gamma))=-\omega(y, z, \gamma)$ for $(y, z, \gamma) \in \mathscr{T}_{n, k}^{(1)}$,

(3) $\Psi_{\mathscr{G}_{n, k}^{(1)}}(\mathbf{x})=0$.

Proof. (1) Elements $y, z$ have different colours in $\mathscr{T}_{n, k}^{(1)}$, so $\psi$ has no fixed points. Moreover

$$
\psi(\psi(y, z, \gamma))=\psi(z, y, \gamma)=(y, z, \gamma),
$$

and the result follows.

(2) If element $y$ is red in $\gamma$, then $z$ is blue in $\gamma$, so $\omega(y, z, \gamma)=w t(\gamma)$ and $\omega(\psi(y, z, \gamma))=\omega(z, y, \gamma)=-w t(\gamma)$. If element $y$ is blue in $\gamma$, then $z$ is red in $\gamma$, so $\omega(y, z, \gamma)=-w t(\gamma)$ and $\omega(\psi(y, z, \gamma))=\omega(z, y, \gamma)=w t(\gamma)$. Thus in either of the two possible cases $\omega(\psi(y, z, \gamma))=-\omega(y, z, \gamma)$.

(3) From (1) and (2) we obtain $\Psi_{\mathscr{F}_{n, k}^{(1)}}(\mathbf{x})=-\Psi_{\mathscr{F}_{n, k}^{(1)}}(\mathbf{x})$ and the result follows.

For the sign-reversing involution $\eta$ on $\mathscr{S}_{n, k}^{(1)}$ we define $L(\rho)$, for $\rho \in \mathscr{A}_{n, k}$, to be the first blue element encountered on the max-cycle of $\rho$ after element $n$ (by construction, any red elements on the max-cycle occur between $n$ and $L(\rho))$. If there are no blue elements on the max-cycle of $\rho$, we define $L(\rho)=0$.

To define $\eta$, let $(u, v, \rho) \in \mathscr{S}_{n, k}^{(1)}$ and assume $u$ is a red element in $\rho$ and $v$ is a blue element in $\rho$ (otherwise exchange $u$ and $v$ ). Then

Case 1. If $L(\rho) \neq 0$ and $v \neq L(\rho)$, then

$$
\eta(u, v, \rho)=(u, L(\rho),(u, L(\rho))(u, v) \rho) .
$$


Case 2. Otherwise, if $L(\rho)=0$ or $v=L(\rho)$, then

$$
\eta(u, v, \rho)=(u, v,(u, v)(u, n)(u, v) \rho(u, n)) \text {. }
$$

For example, with $n=9, k=5, \rho=\left(9,[4]_{R},[7,3]_{B}\right)(1,8,5)_{B}(2,6)_{R}$, so $L(\rho)=7$, we

$$
\begin{aligned}
& \text { A1: } \eta(4,3, \rho)=\left(4,7,\left(9,[3]_{B}\right)(7)_{B}(1,8,5)_{B}(4)_{R}(2,6)_{R}\right), \\
& \text { A2: } \eta(6,3, \rho)=\left(6,7,\left(9,[4,6,2]_{R},[3]_{B}\right)(7)_{B}(1,8,5)_{B}\right), \\
& \text { A3: } \eta(6,8, \rho)=\left(6,7,\left(9,[4,6,2]_{R},[8,5,1,7,3]_{B}\right)\right), \\
& \text { A4: } \eta(4,8, \rho)=\left(4,7,\left(9,[8,5,1,7,3]_{B}\right)(4)_{R}(2,6)_{R}\right), \\
& \text { A5: } \eta(4,7, \rho)=\left(4,7,(9)(3,7)_{B}(1,8,5)_{B}(4)_{R}(2,6)_{R}\right), \\
& \text { A6: } \eta(2,7, \rho)=\left(2,7,\left(9,[6,2,4]_{R}\right)(3,7)_{B}(1,8,5)_{B}\right) .
\end{aligned}
$$

To prove that $\eta$ is a sign-reversing involution, it will be helpful to characterize properly painted permutations $\rho \in \mathscr{A}_{n, k}$ among painted permutations by partitioning $\mathscr{A}_{n, k}$ into two subsets $\mathscr{A}_{n, k}^{(i)}$ for $i=1,2$. Let the image of element $i$ in $\rho$ be $\rho(i)$. Then $\mathscr{A}_{n, k}^{(1)}$ consists of all painted permutations on $\{1, \ldots, n\}$ with $k$ blue elements, in which $\rho(j)=n$ for exactly one blue element $j$ and either $\rho(n)$ is blue or $\rho(n)$ is red and $\rho(j)$ is blue for exactly one red element $j$ (in which case $j=\rho^{-1}(L(\rho))$ ). In every other case, $\rho(j)$ and $j$ have the same colour. (Thus $\mathscr{A}_{n, k}^{(!)}$consists of the properly painted permutations with at least one blue element on the max-cycle.) Similarly $\mathscr{A}_{n, k}^{(2)}$ consists of all painted permutations on $\{1, \ldots, n\}$ with $k$ blue elements, in which either $\rho(n)=n$ or $\rho(n)$ is red and $\rho(j)=n$ for exactly one red element $j$. In every other case $\rho(j)$ and $j$ have the same colour. (Thus $\mathscr{A}_{n, k}^{(2)}$ consists of the properly painted permutations with no blue elements on the max-cycle.)

Using this characterization of the elements of $\mathscr{A}_{n, k}$ we can prove that $\eta$ has the desired properties (it may help to first verify (1) and (2) for the six examples given above).

Theorem 4.4. For $n>k \geq 0$,

(1) $\eta$ is a fixed point-free involution of $\mathscr{S}_{n, k}^{(1)}$,

(2) $\xi(\eta(u, v, \rho))=-\xi(u, v, \rho)$ for $(u, v, \rho) \in \mathscr{S}_{n, k}^{(1)}$,

(3) $\Omega_{\mathscr{S}_{n, k}^{(1)}}(\mathbf{x})=0$.

Proof. Let $\eta(u, v, \rho)=\left(u^{\prime}, v^{\prime}, \rho^{\prime}\right)$.

(1) In Case 1 (e.g., A1, A2, A3, A4), we must have $\rho \in \mathscr{A}_{n, k}^{(1)}$, and calculate that

$$
\rho^{\prime}\left(\rho^{-1}(L(\rho))\right)=u, \quad \rho^{\prime}\left(\rho^{-1}(u)\right)=v, \quad \rho^{\prime}\left(\rho^{-1}(v)\right)=L(\rho)
$$

and $\rho^{\prime}(j)=\rho(j)$ for all other $j=1, \ldots, n$. But $u$ is red and $v, L(\rho)$ are blue. Moreover $\rho^{-1}(v)$ is blue and neither of $\rho^{-1}(u)$ or $\rho^{-1}(L(\rho))$ is blue. Thus $L\left(\rho^{\prime}\right)=v$, and in all cases we can verify that $\left(u^{\prime}, v^{\prime}, \rho^{\prime}\right)$ satisfies Case 1.

Now $v^{\prime} \neq v$ so there are no fixed points for $\eta$ in Case 1. Moreover,

$$
\begin{aligned}
\eta(\eta(u, v, \rho)) & =\eta\left(u^{\prime}, v^{\prime}, \rho^{\prime}\right)=\left(u^{\prime}, L\left(\rho^{\prime}\right),\left(u^{\prime},\left(L\left(\rho^{\prime}\right)\right)\left(u^{\prime}, v^{\prime}\right) \rho^{\prime}\right)\right. \\
& =(u, v,(u, v)(u, L(\rho))(u, L(\rho))(u, v) \rho)=(u, v, \rho)
\end{aligned}
$$

and in this case $\eta$ is a fixed-point-free involution. 
In Case 2, either $\rho \in \mathscr{A}_{n, k}^{(2)}$ (so $L(\rho)=0$ ) or $\rho \in \mathscr{A}_{n, k}^{(1)}$ with $v=L(\rho)$ (e.g., A5, A6), and we calculate that

$$
\rho^{\prime}\left(\rho^{-1}(n)\right)=v, \quad \rho^{\prime}\left(\rho^{-1}(v)\right)=n, \quad \rho^{\prime}(n)=\rho(u), \quad \rho^{\prime}(u)=\rho(n)
$$

and $\rho^{\prime}(j)=\rho(j)$ for all other $j=1, \ldots, n$. But $u$ is red and $v$ is blue. Moreover if $L(\rho)=0$ then $\rho^{-1}(v)$ is blue and none of $\rho(n), \rho^{-1}(n), \rho(u)$ is blue. If $L(\rho)=v$ then $\rho^{-1}(n)$ is blue and either $\rho(n), \rho^{-1}(v)$ are red with $\rho(u)$ red or $\rho(u)=v$, or $\rho(n)=v$ with $\rho(u)$ red. In all cases we can verify that $\left(u^{\prime}, v^{\prime}, \rho^{\prime}\right)$ satisfies Case 2 .

Furthermore, if $\rho \in \mathscr{A}_{n, k}^{(1)}$ then $\rho^{\prime} \in \mathscr{A}_{n, k}^{(2)}$ and if $\rho \in \mathscr{A}_{n, k}^{(2)}$ then $\rho^{\prime} \in \mathscr{A}_{n, k}^{(1)}$, so $\eta$ has no fixed points in Case 2. Moreover,

$$
\begin{aligned}
& \eta(\eta(u, v, \rho))=\eta\left(u^{\prime}, v^{\prime}, \rho^{\prime}\right)=\left(u^{\prime}, v^{\prime},\left(u^{\prime}, v^{\prime}\right)\left(u^{\prime}, n\right)\left(u^{\prime}, v^{\prime}\right) \rho^{\prime}\left(u^{\prime}, n\right)\right) \\
& \quad=\left(u^{\prime}, v^{\prime},\left(u^{\prime}, v^{\prime}\right)\left(u^{\prime}, n\right)\left(u^{\prime}, v^{\prime}\right)(u, v)(u, n)(u, v) \rho(u, n)\left(u^{\prime}, n\right)\right) \\
& \quad=(u, v, \rho) \quad \text { since } u^{\prime}=u, v^{\prime}=v
\end{aligned}
$$

and in this case also $\eta$ is a fixed-point-free involution.

(2) In Case 1,

$$
\left(u^{\prime}, v^{\prime}\right) \rho^{\prime}=(u, L(\rho))(u, L(\rho))(u, v) \rho=(u, v) \rho
$$

and in Case 2,

$$
\left(u^{\prime}, v^{\prime}\right) \rho^{\prime}=(u, v)(u, v)(u, n)(u, v) \rho(u, n) \doteq(u, n)(u, v) \rho(u, n) .
$$

But $(u, n)(u, v) \rho(u, n)$ is conjugate to $(u, v) \rho$, so in both cases we have

$$
d\left(\left(u^{\prime}, v^{\prime}\right) \rho^{\prime}\right)=d((u, v) \rho) .
$$

In both cases, if $v$ lies on a cycle with all elements blue in $\rho$ (e.g., A3, A4), then the elements on that cycle join the max-cycle in $\rho^{\prime}$, and $B\left(\rho^{\prime}\right)=B(\rho)-1$. If $v$ lies on the max-cycle of $\rho$ (e.g., A1, A2, A5, A6), then a new cycle containing $v$, with all elements blue, is created in $\rho^{\prime}$, and $B\left(\rho^{\prime}\right)=B(\rho)+1$. Thus in all cases

$$
(-1)^{B\left(\rho^{\prime}\right)}=-(-1)^{B(\rho)}
$$

and the result follows.

(3) The results in (1) and (2) demonstrate that $\Omega_{\mathscr{S}_{n, k}^{(1)}}(\mathbf{x})=-\Omega_{\mathscr{S}_{n, k}^{(1)}}(\mathbf{x})$ and the result follows immediately.

To define the final mapping $\phi$, let $(u, v, \rho) \in \mathscr{S}_{n, k}^{(2)}$ and define $\phi(u, v, \rho)=$ $(y, z, \gamma)$ where

$$
y= \begin{cases}v & \text { if } u=n \\ u & \text { otherwise }\end{cases}
$$

(ii) $z=v$,

(iii)

$$
\gamma= \begin{cases}\rho(u, v) & \text { if either } u=n, v \text { red or } u \text { red, } v=n \\ (u, v) \rho & \text { otherwise }\end{cases}
$$


For example, with $n=9, k=5, \rho=\left(9,[4]_{R},[7,3]_{B}\right)(1,8,5)_{B}(2,6)_{R}$, we obtain

$$
\begin{aligned}
& \text { B1: } \phi(6,2, \rho)=\left(6,2,\left(9,[4]_{R},[7,3]_{B}\right)(1,8,5)_{B}(2)_{R}(6)_{R}\right), \\
& \text { B2: } \phi(7,8, \rho)=\left(7,8,\left(9,[4]_{R},[8,5,1,7,3]_{B}\right)(2,6)_{R}\right), \\
& \text { B3: } \rho(2,9, \rho)=\left(2,9,\left(9,[6,2,4]_{R},[7,3]_{B}\right)(1,8,5)_{B}\right), \\
& \text { B4: } \rho(3,9, \rho)=\left(3,9,\left(9,[4]_{R},[7]_{B}\right)(3)_{B}(1,8,5)_{B}(2,6)_{R}\right), \\
& \text { B5: } \phi(9,4, \rho)=\left(4,4,\left(9,[7,3]_{B}\right)(1,8,5)_{B}(4)_{R}(2,6)_{R}\right), \\
& \text { B6: } \phi(9,5, \rho)=\left(5,5,\left(9,[4]_{R},[7,3,5,1,8]_{B}\right)(2,6)_{R}\right) .
\end{aligned}
$$

We now prove that $\phi$ has the required properties.

Theorem 4.5. For $n>k \geq 0$

(1) $\phi$ is a bijection from $\mathscr{S}_{n, k}^{(2)}$ to $\mathscr{T}_{n, k}^{(2)}$,

(2) $\omega(\phi(u, v, \rho))=\xi(u, v, \rho)$ for $(u, v, \rho) \in \mathscr{S}_{n, k}^{(2)}$,

(3) $\Omega_{\mathscr{S}_{n, k}^{(2)}}(\mathbf{x})=\Psi_{\mathscr{F}_{n, k}^{(2)}}(\mathbf{x})$.

Proof. Let $\phi(u, v, \rho)=(y, z, \gamma)$ for $(u, v, \rho) \in \mathscr{S}_{n, k}^{(2)}$.

(1) Since $u, v$ are not of different colours, then $y, z$ are not of different colours. Moreover $1 \leq z \leq n$ because $1 \leq v \leq n$ and $1 \leq y \leq n-1$ because $u \neq v$ and $1 \leq u \leq n$. Now if $\gamma=\rho(u, v)$ (e.g. B3, B5) then

$$
\gamma(v)=\rho(u), \quad \gamma(u)=\rho(v)
$$

and $\gamma(j)=\rho(j)$ for all other $j$. If $\gamma=(u, v) \rho$ (e.g., B1, B2, B4, B6) then

$$
\gamma\left(\rho^{-1}(u)\right)=v, \quad \gamma\left(\rho^{-1}(v)\right)=u
$$

and $\gamma(j)=\rho(j)$ for all other $j$. In both cases it is straightforward to verify that $\gamma \in \mathscr{A}_{n, k}$, so $(y, z, \gamma) \in \mathscr{T}_{n, k}^{(2)}$ and $\phi$ is an injection. But $\phi$ is a bijection since we can compute its inverse: if $(y, z, \gamma) \in \mathscr{T}_{n, k}^{(2)}$ then

$$
\phi^{-1}(y, z, \gamma)=(u, v, \rho)
$$

where

(i)

$$
u= \begin{cases}n & \text { if } y=z \\ y & \text { otherwise }\end{cases}
$$

(ii) $v=z$,

(iii)

$$
\rho= \begin{cases}\gamma(u, v) & \text { if either } y \text { red, } z=n \text { or } z=y, \\ (u, v) \gamma & \text { otherwise. }\end{cases}
$$

Again it is straightforward to verify that $1 \leq u \neq v \leq n$ and $\rho \in \mathscr{A}_{n, k}$ so $(u, v, \rho) \in \mathscr{S}_{n, k}^{(2)}$. The result follows.

(2) We have

and

$$
\xi(u, v, \rho)=(-1)^{B(\rho)} p_{d((u, v) \rho)}
$$

$$
\omega(y, z, \gamma)= \begin{cases}(-1)^{B(\gamma)} p_{d(\gamma)} & \text { if } y \text { is red in } \gamma, \\ -(-1)^{B(\gamma)} p_{d(\gamma)} & \text { if } y \text { is blue in } \gamma .\end{cases}
$$


Now $\gamma=(u, v) \rho$ or $\rho(u, v)$ and $(u, v) \rho$ is conjugate to $\rho(u, v)$, so in both cases we have

$$
d((u, v) \rho)=d(\gamma) .
$$

Moreover, if neither of $u, v$ is red, then $\gamma=(u, v) \rho$ has one more cycle with all elements blue than $\rho$ if $u, v$ are on the same cycle of $\rho$ (e.g., B4) and one less cycle if $u, v$ are on different cycles (e.g., B2, B6). But this means that $y$ is blue, so in this case

$$
(-1)^{B(\rho)}=-(-1)^{B(\gamma)} \text {. }
$$

If neither of $u, v$ is blue (e.g., B1, B3, B5), then $(u, v) \rho$ and $\rho(u, v)$ have the same number of cycles with all elements blue as $\rho$, and $y$ is red, so

$$
(-1)^{B(\rho)}=(-1)^{B(\gamma)}
$$

whether $\gamma=\rho(u, v)$ or $(u, v) \rho$. The result follows immediately.

(3) This follows directly from (1) and (2).

We now have completed all the pieces for a combinatorial derivation of Theorem 3.2 .

Combinatorial proof of Theorem 3.2.

$$
\begin{aligned}
2 \Delta A_{n, k} & =\Omega_{\mathscr{S}_{n, k}}(\mathbf{x}), \quad \text { from Proposition 4.2(1) } \\
& =\Omega_{\mathscr{S}_{n, k}^{(1)}}(\mathbf{x})+\Omega_{\mathscr{S}_{n, k}^{(2)}}(\mathbf{x}) \\
& =\Omega_{\mathscr{S}_{n, k}^{(2)}}(\mathbf{x}), \quad \text { from Theorem 4.4(3) } \\
& =\Omega_{\mathscr{F}_{n, k}^{(2)}}(\mathbf{x}), \quad \text { from Theorem 4.5(3) } \\
& =\Omega_{\mathscr{G}_{n, k}^{(1)}}(\mathbf{x})+\Omega_{\mathscr{F}_{n, k}^{(2)}}(\mathbf{x}), \quad \text { from Theorem 4.3(3) } \\
& =\Omega_{\mathscr{F}_{n, k}}(\mathbf{x}) \\
& =2\left\{\left(\begin{array}{l}
n \\
2
\end{array}\right)-n k\right\} A_{n, k}, \quad \text { from Proposition 4.2(2). }
\end{aligned}
$$

This proves Theorem 3.2(2) (multiplied by 2). Theorem 3.2(1) follows immediately by multiplying on both sides by $r^{n-1-k} b^{k} t^{n} / n$ ! and summing over $n>k \geq 0$.

The author has been unable to choose the pairs $u, v$ and $y, z$ canonically to remove the factor of 2 on both sides.

In the next section, the combinatorial steps in this proof are iterated to yield a combinatorial proof of the main result.

\section{THE COMBINATORICS OF MULTIPLYING TRANSPOSITIONS}

We now extend the combinatorial material in $\S 4$ to multiplication by $m$ transpositions. To do this, define sets $\mathscr{S}_{n, k, m}$ and $\mathscr{T}_{n, k, m}$ by

$$
\begin{aligned}
& \mathscr{S}_{n, k, m}=\left\{\left(\left(u_{1}, v_{1}\right), \ldots,\left(u_{m}, v_{m}\right), \rho\right) \mid 1 \leq u_{i} \neq v_{i} \leq n,\right. \\
& \left.i=1, \ldots, m, \rho \in \mathscr{A}_{n, k}\right\} \text {, } \\
& \mathscr{T}_{n, k, m}=\left\{\left(\left(y_{1}, z_{1}\right), \ldots,\left(y_{m}, z_{m}\right), \gamma\right) \mid 1 \leq y_{i} \leq n-1,\right. \\
& \left.1 \leq z_{i} \leq n, i=1, \ldots, m, \gamma \in \mathscr{A}_{n, k}\right\} \text {. }
\end{aligned}
$$


For $\left(\left(u_{1}, v_{1}\right), \ldots,\left(u_{m}, v_{m}\right), \rho\right) \in \mathscr{S}_{n, k, m}$, define the weight function

$$
\xi^{(m)}\left(\left(u_{1}, v_{1}\right), \ldots,\left(u_{m}, v_{m}\right), \rho\right)=(-1)^{B(\rho)} p_{d\left(\left(u_{1}, v_{1}\right) \cdots\left(u_{m}, v_{m}\right) \rho\right)} \cdot
$$

For $\left(\left(y_{1}, z_{1}\right), \ldots,\left(y_{m}, z_{m}\right), \gamma\right) \in \mathscr{T}_{n, k, m}$, let $N\left(y_{1}, \ldots, y_{m} ; \gamma\right)$ be the number of $y_{i}, i=1, \ldots, n$, for which element $y_{i}$ is blue in $\gamma$, and define the weight function

$$
\omega^{(m)}\left(\left(y_{1}, z_{1}\right), \ldots,\left(y_{m}, z_{m}\right), \gamma\right)=(-1)^{N\left(y_{1}, \ldots, y_{m} ; \gamma\right)}(-1)^{B(\gamma)} p_{d(\gamma)} .
$$

Generating functions with respect to these weight functions are given by

$$
\begin{array}{ll}
\Omega_{\mathscr{S}}^{(m)}(\mathbf{x})=\sum_{s \in \mathscr{S}} \xi^{(m)}(s) & \text { for } \mathscr{S} \subseteq \mathscr{S}_{n, k, m}, \\
\Psi_{\mathscr{T}}^{(m)}(\mathbf{x})=\sum_{t \in T} \omega^{(m)}(t) & \text { for } \mathscr{T} \subseteq \mathscr{T}_{n, k, m} .
\end{array}
$$

The extension of Proposition 4.2 is immediate.

Proposition 5.1. For $n>k \geq 0, m \geq 0$ we have

(1) $\Omega_{\mathscr{S}_{n, k, m}^{(m)}}^{(m)}(\mathbf{x})=2^{m} \Delta^{m} A_{n, k}$,

(2) $\Psi_{\mathscr{T}_{n, k, m}}^{(m)}(\mathbf{x})=2^{m}\left\{\left(\begin{array}{l}n \\ 2\end{array}\right)-n k\right\}^{m} A_{n, k}$.

We now proceed as in $\S 4$, to deduce a combinatorial proof of the main result by giving a direct combinatorial proof that

$$
\Omega_{\mathscr{S}_{n, k, m}}^{(m)}(\mathbf{x})=\Psi_{\mathscr{T}_{n, k, m}}^{(m)}(\mathbf{x}) .
$$

This proof of (5.1) proceeds by partitioning $\mathscr{S}_{n, k, m}$ into subsets $\mathscr{S}_{n, k, m}^{(1)}$ and $\mathscr{S}_{n, k, m}^{(2)}$, and $\mathscr{T}_{n, k, m}$ into subsets $\mathscr{T}_{n, k, m}^{(1)}$ and $\mathscr{T}_{n, k, m}^{(2)}$. We give sign-reversing involutions $\eta^{(m)}$ on $\mathscr{S}_{n, k, m}^{(1)}$ and $\psi^{(m)}$ on $\mathscr{T}_{n, k, m}^{(1)}$, proving that $\Omega_{\mathscr{S}_{n, k, m}^{(1)}}^{(m)}(\mathbf{x})=0$ and $\Psi_{\mathscr{G}_{n, k, m}^{(1)}}^{(m)}(\mathbf{x})=0$. Then we give a weight-preserving bijection $\phi^{(m)}$ between $\mathscr{S}_{n, k, m}^{(2)}$ and $\mathscr{T}_{n, k, m}^{(2)}$, proving that $\Omega_{\mathscr{S}_{n, k, m}^{(2)}}^{(m)}=\Psi_{\mathscr{G}_{n, k, m}^{(2)}}^{(m)}(\mathbf{x})$, and hence arriving at (5.1).

The sets $\mathscr{S}_{n, k, m}^{(i)}, \mathscr{T}_{n, k, m}^{(i)}, i=1,2$, and mappings $\eta^{(m)}, \psi^{(m)}, \phi^{(m)}$ are defined in terms of the sets $\mathscr{S}_{n, k}^{(i)}, \mathscr{T}_{n, k}^{(i)}, i=1,2$, and mappings $\eta, \psi, \phi$ of $\S 4$, as follows: First let

$\mathscr{S}_{n, k, m}^{(1)}=\left\{\left(\left(u_{1}, v_{1}\right), \ldots,\left(u_{m}, v_{m}\right), \rho\right) \in \mathscr{S}_{n, k, m} \mid\right.$

element $u_{i}, v_{i}$ have different colours in $\rho$ for some $i=1, \ldots, m$ \},

and let $\eta^{(m)}$ act on $\left(\left(u_{1}, v_{1}\right), \ldots,\left(u_{m}, v_{m}\right), \rho\right) \in \mathscr{S}_{n, k, m}^{(1)}$ by replacing $\left(u_{i}, v_{i}\right.$, $\rho)$ by $\left(u_{i}^{\prime}, v_{i}^{\prime}, \rho^{\prime}\right)=\eta\left(u_{i}, v_{i}, \rho\right)$ and leaving all other $u_{j}, v_{j}$ unchanged, where $i$ is the minimum choice such that $u_{i}, v_{i}$ have different colours in $\rho$. Since all elements have the same colour in $\rho$ as in $\rho^{\prime}$, the proof of Theorem 4.4 immediately yields a proof that $\eta^{(m)}$ is a fixed-point-free sign-reversing involution on $\mathscr{S}_{n, k, m}^{(1)}$, and hence we have a combinatorial proof that

$$
\Omega_{S_{n, k, m}^{(1)}}^{(m)}(\mathbf{x})=0
$$


Next consider

$$
\begin{aligned}
\mathscr{T}_{n, k, m}^{(1)}= & \left\{\left(\left(y_{1}, z_{1}\right), \ldots,\left(y_{m}, z_{m}\right), \gamma\right) \in \mathscr{T}_{n, k, m} \mid\right. \\
& \left.\quad \text { elements } y_{i}, z_{i} \text { have different colours in } \gamma \text { for some } i=1, \ldots, m\right\},
\end{aligned}
$$

and let $\psi^{(m)}$ act on $\left(\left(y_{1}, z_{1}\right), \ldots,\left(y_{m}, z_{m}\right), \gamma\right) \in \mathscr{T}_{n, k, m}^{(1)}$ by interchanging $y_{i}$ and $z_{i}$ for the minimum $i=1, \ldots, m$ such that $y_{i}$ and $z_{i}$ have different colours (thus replacing $\left(y_{i}, z_{i}, \gamma\right)$ by $\psi\left(y_{i}, z_{i}, \gamma\right)$ ). Then we follow the proof of Theorem 4.3 to prove that $\psi^{(m)}$ is a fixed-point-free sign-reversing involution on $\mathscr{G}_{n, k, m}^{(1)}$, and hence we have a combinatorial proof that

$$
\Psi_{\mathscr{T}_{n, k, m}^{(m)}}^{(m)}(\mathbf{x})=0
$$

Finally we describe the mapping $\phi^{(m)}$ from $\mathscr{S}_{n, k, m}^{(2)}$ to $\mathscr{T}_{n, k, m}^{(2)}$ (the complements of $\mathscr{S}_{n, k, m}^{(1)}$ and $\mathscr{T}_{n, k, m}^{(1)}$ in $\mathscr{S}_{n, k, m}$ and $\left.\mathscr{T}_{n, k, m}\right)$. Consider $s=$ $\left(\left(u_{1}, v_{1}\right), \ldots,\left(u_{m}, v_{m}\right), \rho\right) \in \mathscr{S}_{n, k, m}^{(2)}$ and let $\gamma_{m}=\rho$. In succession, as $j=0,1, \ldots, m-1$, let

$$
\phi\left(u_{m-j}, v_{m-j}, \gamma_{m-j}\right)=\left(y_{m-j}, z_{m-j}, \gamma_{m-j-1}\right) .
$$

This is well-defined since all elements have the same colour in $\gamma_{m-j}$ as in $\rho$ for all $j$, so $\left(u_{m-j}, v_{m-j}, \gamma_{m-j}\right) \in \mathscr{S}_{n, k}^{(2)}$ for all $j$. Then

$$
\phi^{(m)}(s)=\left(\left(y_{1}, z_{1}\right), \ldots,\left(y_{m}, z_{m}\right), \gamma_{0}\right) .
$$

For example, when $n=9, k=5, m=4$,

$$
\begin{array}{r}
\phi^{(4)}\left((8,9),(5,3),(9,7),(2,9),\left(9,[4]_{R},[7,3]_{B}\right)(1,8,5)_{B}(2,6)_{R}\right) \\
\quad=\left((8,9),(5,3),(7,7),(2,9),\left(9,[6,2,4]_{R},[8,3,7,5,1]_{B}\right)\right) .
\end{array}
$$

The proof of Theorem 4.5 allows us to establish immediately that $\phi^{(m)}$ is a weight-preserving bijection from $\mathscr{S}_{n, k, m}^{(2)}$ to $\mathscr{T}_{n, k, m}^{(2)}$, and hence gives a combinatorial proof that

$$
\Omega_{\mathscr{S}_{n, k, m}^{(2)}}^{(m)}(\mathbf{x})=\Psi_{\mathscr{T}_{n, k, m}^{(2)}}^{(m)}(\mathbf{x}) .
$$

Combining (5.2), (5.3), (5.4) we have a direct combinatorial proof of (5.1) and hence, via Proposition 5.1, of Theorem 2.1, the main result (multiplied by $\left.2^{m}\right)$.

One more combinatorial step is needed to obtain a direct combinatorial proof of Theorem 1.1. This is to account for the application of Proposition 1.2(5). Consider $\rho \in \dot{U}_{k} \mathscr{A}_{n, k}$. If $d(\rho) \neq(n)$ find the largest element in $\{1, \ldots, n-1\}$ not on the max-cycle and switch the colour of the cycle containing that element, to obtain $\zeta(\rho)=\rho^{\prime} \in \dot{U}_{k} \mathscr{A}_{n, k}$ with $d\left(\rho^{\prime}\right) \neq(n)$. But $B\left(\rho^{\prime}\right)=B(\rho) \pm 1$ and $d(\rho)=d\left(\rho^{\prime}\right)$ so $\Phi(\rho)=-\Phi\left(\rho^{\prime}\right)$. Moreover $\rho \neq \rho^{\prime}$ and $\zeta(\zeta(\rho))=\rho$ so $\zeta$ is a fixed-point-free sign-reversing involution on $\left\{\rho \in \bigcup_{k} \mathscr{A}_{n, k} \mid d(\rho) \neq(n)\right\}$, and we have a combinatorial proof that

$$
\sum_{k=0}^{n} \mathscr{A}_{n, k}=\sum_{k=0}^{n-1} \sum_{\rho \in \mathscr{A}_{n, k}} \Phi(\rho)=\alpha \rho_{n}
$$


where $\alpha$ is the number of ways of properly painting an $n$-cycle, so $\alpha=n$.

We now give a combinatorial proof of Theorem 1.1 (multiplied by $n ! 2^{m}$ ).

Combinatorial proof of Theorem 1.1. On the RHS, after multiplying by $n ! 2^{m}$ we have

$$
\sum_{k=0}^{n-1}\{(n-1-2 k) n\}^{m}\left(\begin{array}{c}
n-1 \\
k
\end{array}\right)(-1)^{k}=\left[p_{1}^{n}\right] \sum_{k=0}^{n-1} \Psi_{\mathscr{S}_{n, k, m}}^{(m)}(\mathbf{x}),
$$

and on the LHS we have

$$
2^{m} n ! c_{\alpha_{1}, \ldots, \alpha_{m}}^{(n)}=\left[p_{1}^{n}\right] \sum_{k=0}^{n-1} \Omega_{\mathscr{S}_{n, k, m}}^{(m)}(\mathbf{x}) .
$$

The combinatorial proof proceeds as follows: For each $s=\left(\left(u_{1}, v_{1}\right), \ldots\right.$, $\left.\left.\left(u_{m}, v_{m}\right), \rho\right)\right) \in \dot{\bigcup}_{k} \mathscr{S}_{n, k, m}^{(2)}$ with $d(\rho)=(n)$ and $d\left(\left(u_{1}, v_{1}\right) \cdots\left(u_{m}, v_{m}\right) \rho\right)=$ $1^{n}$, find $\phi^{(m)}(s)=t=\left(\left(y_{1}, z_{1}\right), \ldots,\left(y_{m}, z_{m}\right), \gamma\right) \in \dot{\bigcup}_{k} \mathscr{T}_{n, k, m}^{(2)}$, with $d(\gamma)=$ $1^{n}$.

The contributions of $s$ with $d(\rho) \neq(n)$ and $d\left(\left(u_{1}, v_{1}\right) \cdots\left(u_{m}, v_{m}\right) \rho\right)=1^{n}$ are cancelled by application of $\zeta$.

(The contributions of elements of $\mathscr{T}_{n, k, m}^{(1)}$ and $\mathscr{S}_{n, k, m}^{(1)}$ are again cancelled by $\eta^{(m)}$ and $\psi^{(m)}$, respectively.)

The author has been unable to remove the factor of $2^{m}$ (due to working with ordered pairs as transpositions) nor the factor $n$ ! (due to working with all properly painted $n$-cycles). In the special case $m=n-1$, the relationship to the combinatorial proofs of Moszkowski [18] or Goulden and Pepper [10] is quite unclear, especially because the bijection in this paper is between two sets with alternating weights, and not directly involving labelled trees at all.

\section{ACKNOWLEDGMENTS}

This work was supported by grant A8907 from the National Sciences and Engineering Research Council of Canada.

\section{REFERENCES}

1. F. Bédard and A. Goupil, The poset of conjugacy classes and decomposition of products in the symmetric group, Canad. Math. Bull. 35 (1992), 152-160.

2. E. A. Bertram and V. K. Wei, Decomposing a permutation into two large cycles: An enumeration, SIAM J. Algebraic Discrete Meth. 1 (1980), 450-461.

3. G. Boccara, Nombre de représentations d'une permutation comme produit de deux cycles de longueurs données, Discrete Math. 29 (1980), 105-134.

4. M. Burrow, Representation theory of finite groups, Academic Press, New York, 1965.

5. A. Cayley, A theorem on trees, Quart. J. Math. Oxford 23 (1889), 376-378.

6. J. Dénes, The representation of a permutation as the product of a minimal number of transpositions and its connection with the theory of graphs, Publ. Math. Inst. Hungar. Acad. Sci. 4 (1959), 63-70.

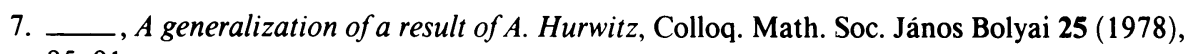
85-91.

8. I. P. Goulden and D. M. Jackson, Combinatorial enumeration, Wiley-Interscience, New York, 1983. 
9. for the symmetric group, European J. Combin. 13 (1992), 357-365.

10. I. P. Goulden and S. Pepper, Labelled trees and factorizations of a cycle into transpositions, Discrete Math. 113 (1993), 263-268.

11. A. Hurwitz, Über Riemannsche Flächen mit gegebenen Verzweigungspunkten, Math. Ann. 39 (1891), 1-61.

12. _ـ Über die Anzahl der Riemannschen Flächen mit gegebenen Verzweigungspunkten, Math. Ann. 55 (1902), 53-66.

13. D. M. Jackson, Counting cycles in permutations by group characters, with an application to a combinatorial problem, Trans. Amer. Math. Soc. 299 (1987), 785-801.

14. __ Counting semiregular permutations which are products of a full cycle and an involution, Trans. Amer. Math. Soc. 305 (1988), 317-331.

15. - Some problems associated with products of conjugacy classes of the symmetric group, J. Combin. Theory Ser. A 49 (1988), 363-369.

16. D. E. Littlewood, The theory of group characters, 2nd ed., Oxford Univ. Press, London, 1950.

17. I. G. Macdonald, Symmetric functions and Hall polynomials, Clarendon Press, Oxford, 1979.

18. P. Moszkowski, $A$ solution to a problem of Dénes: a bijection between trees and factorizations of cyclic permutations, European J. Combin. 10 (1989), 13-16.

19. R. P. Stanley, Factorization of a permutation into n-cycles, Discrete Math. 37 (1981), 255-262.

20. D. W. Walkup, How may ways can a permutation be factored into two n-cycles?, Discrete Math. 28 (1979), 315-319.

Department of Combinatorics and Optimization, University of Waterloo, Waterloo, ONTARIO, N2L 3G1 CANADA

E-mail address: ipgoulden@math. uwaterloo.ca 